Article

\title{
Incipient Fault Feature Extraction of Rolling Bearings Using Autocorrelation Function Impulse Harmonic to Noise Ratio Index Based SVD and Teager Energy Operator
}

\author{
Kai Zheng ${ }^{1}$ (i), Tianliang Li ${ }^{2}{ }^{(1)}$, Bin Zhang ${ }^{1}$, Yi Zhang ${ }^{1}$, Jiufei Luo ${ }^{1}$ and Xiangyu Zhou ${ }^{1, *}$ \\ 1 School of Advanced Manufacturing Engineering, Chongqing University of Posts and Telecommunications, \\ Chongqing 400065, China; zhengkai2001@163.com (K.Z.); zhangbin@cqupt.edu.cn (B.Z.); \\ zhangyi@cqupt.edu.cn (Y.Z.); luojf@cqupt.edu.cn (J.L.) \\ 2 SMRT-NTU Smart Urban Rail Corporate Laboratory, Nanyang Technological University, \\ Singapore 639798, Singapore; tianliangliwhut@sina.com \\ * Correspondence: zhouxy@cqupt.edu.cn; Tel.: +86-187-1628-5005
}

Received: 2 September 2017; Accepted: 25 October 2017; Published: 30 October 2017

\begin{abstract}
The periodic impulse feature is the most typical fault signature of the vibration signal from fault rolling element bearings (REBs). However, it is easily contaminated by noise and interference harmonics. In order to extract the incipient impulse feature from the fault vibration signal, this paper presented an autocorrelation function periodic impulse harmonic to noise ratio (ACFHNR) index based on the SVD-Teager energy operator (TEO) method. Firstly, the Hankel matrix is constructed based on the raw vibration fault signal of rolling bearing, and the SVD method is used to obtain the singular components. Afterwards, the ACFHNR index is employed to measure the abundance of the periodic impulse fault feature for the singular components, and the component with the largest ACFHNR index value is extracted. Moreover, the properties of the ACFHNR index are demonstrated by simulations and the full life cycle of the experiment, showing its superiority over the traditional kurtosis and root mean square (RMS) index for extracting and detecting incipient periodic impulse features. Finally, the Teager energy operator spectrum of the extracted informative signal is gained. The simulation and experimental results indicated that the proposed ACFHNR index based method can effectively detect the incipient fault feature of the rolling bearing, and it shows better performance than the kurtosis and RMS index based methods.
\end{abstract}

Keywords: rolling element bearings (REBs); singular value decomposition (SVD); autocorrelation function impulse harmonic to noise ratio (ACFHNR); teager energy operator (TEO)

\section{Introduction}

Rolling element bearings have been widely used in scenarios such as wind turbines, high-speed railways, and precision machine tools. They generally operate in tough working environments and are easily subject to failures, which may cause machinery to break down and decrease machinery service performance such as manufacturing quality or operation safety [1-4]. When a defect occurs on a rolling bearing surface, the impulses are created in vibration signals $[5,6]$. As a result, the detection of faults in rolling element bearings is mainly achieved by identifying the frequency of the impulses from the signals $[7,8]$. For complicated mechanical systems, the rolling bearing often works in complicated environments, and the vibration signals are easily contaminated by environmental noise and other working parts such as the gearbox (misalignment, unbalance, crack on the rotating shaft, looseness, and distortions). Therefore, their early impulse faults often feature weak and low signal to noise ratios (SNR) $[5,6]$. 
Many advanced signal decomposition methods have been studied for fault feature extraction in the field of rotary machine diagnosis. The typical multicomponent decomposition methods include short-time Fourier transform [9], wavelet transform [10], Hilbert-Huang transform [11], Empirical Mode Decomposition/Ensemble Empirical Mode Decomposition (EMD/EEMD) [12,13], and Local Mean Decomposition (LMD) [14]. Among a wide range of de-noising and signal enhancing methods available in the literature, noise elimination algorithms based on singular value decomposition (SVD) are considerably faster and easier to implement $[5,8]$. Also, it is a non-parametric signal analysis tool, which can be implemented without pre-defined basis functions. Therefore, the SVD method has been employed for fault detection in much research [15-19].

In order to extract the impulse fault feature from the vibration signals of the rolling bearing, it is very important to select the effective singular components for reconstructing a new signal when the SVD method is applied. There are many traditional methods such as the median value of singular values, mean value of singular values, and Difference spectrum of singular values (DSSV) based SVD methods [1]. Among them, the well-established traditional median value of singular values based SVD method and the mean value of singular values based SVD method choose the effective singular components with large singular values. It is believed that the larger singular values can contain much more useful fault features when these methods are applied. However, as the literature $[1,20]$ points out, a larger singular value corresponding to singular components may not contain the informative fault feature. The energy value of the informative fault feature may be very weak. A Difference spectrum of singular values (DSSV) is introduced to capture the abrupt change in the singular values, which reflects the boundary between the signal and the noise, based on the principle that the effective informative singular components can be extracted $[20,21]$. The literature [1] proposed a method to capture the abrupt change based on the correlation coefficient singular value decomposition (CCSVD) method. However, these methods are characterized by capturing the abrupt change and may remove the useful signals and retain the noise signals. As a result, they may not be able to extract the weak fault feature effectively. To solve this problem, one study [5] proposed kurtosis to quantify the impulsive feature of the vibration signal, and the simulation and experiment results indicate that the de-noising method is successful in both the frequency domain and time domain for fault identification. Another study [15] proposed a Periodic Modulation Intensity (PMI) index to select fault features with relatively lower energy levels, and it is robust in the presence of heavy noise and strong random interferences.

From the above analysis, it can be seen that it is vital to select suitable singular components for extracting mechanical fault features using the SVD method. The crucial step is to find an index to measure the abundance of fault information for the singular components. Although kurtosis shows high values for extracting the impulsive feature [22], the background noises and rotating frequency-related interference harmonic components in the collected vibration signal would submerge the periodic impulse fault feature. As a result, the kurtosis value may not be able to reveal the useful information. The root-mean-square (RMS) value of the measured signal is applied in industry to measure the vibration intensity and indicate the incipient defect. However, the RMS value has limited applications because it is not sensitive to defect at its early stage, when the defect contributes little energy [23]. Moreover, these two indicators were originally developed in the field of statistics or information theory, thus they mainly focus on the general statistical distribution of a signal but may ignore the specific characteristics of mechanical signals [15].

Motivated by this, an autocorrelation function impulse harmonic to noise ratio index (ACFHNR) is proposed to measure the richness of the periodic impulse fault feature information in this paper. According to the proposed index, the ACFHNR based SVD-Teager energy operator (TEO) method for extracting the incipient fault feature from the vibration signal of the rolling bearing is presented. This paper is organized as follows: Section 2 introduces the theoretical background, and an ACFHNR index is proposed. Based on the proposed index, a novel method for extracting the incipient impulse fault feature of a rolling bearing using the ACFHNR based SVD-TEO is proposed in Section 3. Section 4 
makes a simulation analysis of the proposed method. In Section 5 , the proposed method is verified by experimental data, and Section 6 makes several conclusions.

\section{Theory Background}

\subsection{The Fundamental of SVD Denoising Method}

SVD is an orthogonal decomposition method. For any real matrix $\mathbf{A} \in \mathbf{R}_{m \times n}$ with rank $L$, it can be decomposed into the product of three matrices [5]: the orthogonal matrix $\mathbf{U} \in \mathbf{R}_{m \times n}$, the diagonal matrix $\mathbf{D}=\left[\operatorname{diag}\left(\sigma_{1}, \sigma_{2} \ldots, \sigma_{l}\right), 0\right]$, and the orthogonal matrix $\mathbf{V} \in \mathbf{R}_{m \times n}$, as follows:

$$
\mathbf{A}=\mathbf{U D V}^{\mathbf{T}}
$$

where $L=\min (m, n), \sigma_{1} \geq \sigma_{2} \ldots \geq \sigma_{l} . \sigma_{i}(i=1,2 \ldots L)$ are the singular values of the matrix $\mathbf{A}$.

There are several steps to denoise the measurement signal with the SVD method. Firstly, the Hankel matrix should be constructed [24-27] as follows:

$$
\mathbf{A}_{m}=\left[\begin{array}{cccc}
x(1) & x(2) & \cdots & x(n) \\
x(2) & x(3) & \cdots & x(n+1) \\
\vdots & \vdots & \ddots & \vdots \\
x(m) & x(m+1) & \cdots & x(N)
\end{array}\right]
$$

where $1<n<N, m=N-n+1$. The parameter $m$ is used to determine the number of decomposed components in SVD, which is usually selected less than $n$ [15].

Secondly, the dynamic propriety of the matrix $\mathbf{A}_{m}$ can be revealed based on the reconstructed attractor in the reconstructed phase space. As a result, the matrix $\mathbf{A}_{m}$ can be expressed as [28]:

$$
\mathbf{A}_{m}=\mathbf{D}+\mathbf{W}
$$

where $\mathbf{D}$ is the smooth signal in the reconstructed phase space and $\mathbf{W}$ is the noise and interference signal in the reconstructed phase space. To denoise the original signal, it is necessary to find the best approximation matrix for matrix $\mathbf{D}$. According to the singular value decomposition principle, the matrix $\mathbf{A}_{m}$ can be decomposed as follows:

$$
\mathbf{A}_{m}=\mathbf{U E V}^{\mathbf{T}}
$$

where $\mathbf{E}=\left[\operatorname{diag}\left(\sigma_{1}, \sigma_{2} \ldots, \sigma_{m}\right), \mathbf{0}\right]$ and $L=\min (m, n), \sigma_{1} \geq \sigma_{2} \ldots \geq \sigma_{l} . \mathbf{U}$ and $\mathbf{V}^{\mathbf{T}}$ are the left and right singular matrixes, respectively, and they satisfied $\mathbf{U}=\left[u_{1}, u_{2} \ldots, u_{m}\right], \mathbf{V}=\left[v_{1}, v_{2} \ldots, v_{n}\right]^{\mathbf{T}}$, where $u_{i}$ is the $i$ th column vector of singular matrix $\mathbf{U}$ and $v_{i}$ is the $i$ th column vector of singular matrix $\mathbf{V}$. As a result, $\mathbf{A}_{m}$ can be computed as:

$$
\mathbf{A}_{m}=\sigma_{1} u_{1} v_{1}+\sigma_{2} u_{2} v_{2}+\ldots+\sigma_{m} u_{m} v_{m}
$$

Finally, according to the singular value decomposition theory and the best approximation theorem of a matrix in the sense of Frobeious norm, it can be obtained that the different kinds of signals are characterized by different singular values [28]. Therefore, by removing the singular values corresponding to the noise components and interference harmonics, we can get the new diagonal matrix $\mathbf{E}^{\prime}=\left[\operatorname{diag}\left(\sigma_{\alpha}, \sigma_{j} \ldots, \sigma_{\beta}\right), 0\right]$. The new Hankel matrix $\mathbf{A}_{m}{ }^{\prime}$ can be computed as:

$$
\mathbf{A}_{m}{ }^{\prime}=\sigma_{\alpha} u_{\alpha} v_{\alpha}+\sigma_{j} u_{j} v_{j}+\ldots+\sigma_{\beta} u_{\beta} v_{\beta}
$$

where the matrix $\mathbf{A}_{m}{ }^{\prime}$ is supposed to be the best approximation of matrix $\mathbf{A}_{m}$. Compared to the original matrix $\mathbf{A}_{m}$, its noise components and interference harmonics are compressed significantly. 
The averaging value of the anti-diagonals element $x_{i}$ of the matrix $\mathbf{A}_{m}{ }^{\prime}$ is calculated, which is regarded as the denoised signal for the original vibration signal [5], as shown in Equation (7):

$$
\mathbf{x}_{i}=\frac{1}{\beta-\alpha+1} \sum_{j=\alpha}^{\beta} \mathbf{A}_{i-j+1, j}
$$

For traditional SVD denoising methods such as the median value of singular values, the mean value of singular values, and DSSV based SVD denoising, the most energic singular components are reserved. However, for the vibration signal of the rolling elements bearing, the incipient impulse fault feature is often submerged by the noise and interference harmonics, and its energy may be very weak. As a result, the traditional energy based evaluation method of SVD denoising could be invalid for extracting the informative impulse fault feature of a rolling bearing. It is important to find a new method for evaluating the fault information of the singular components.

\subsection{Autocorrelation Function Impulse Harmonic to Noise Ratio Index}

When a defect impacts the rolling surface, cyclic impulses are generated. A variety of methods to decompose the fault signal for extracting the weak periodic impulse feature were proposed such as EEMD [12,13], LMD [14], intrinsic characteristic-scale decomposition (ICD) [29]. However, how to choose the sensitive feature components remains a problem to be solved. Many scholars have proposed a series of indexes such as kurtosis [22], approximate entropy [30], Pearson coefficient [13], or a fusion of these parameters [31] to measure the richness of fault feature information in the decomposed components. However, most of these indicators were originally developed in the field of statistics or information theory, thus they mainly focus on the general statistical distribution of a signal, such as its non-Gaussianity and peakedness, but may ignore the specific characteristics of mechanical signals [15]. Therefore, we proposed a new index, which is called the autocorrelation function periodic impulse harmonic to noise ratio index (ACFHNR). It focuses on the detection of the periodic impulse fault features of the rolling bearing.

In the bearing fault signal, the effective feature information is often contaminated by noise. Generally, it is assumed that the noise in the fault signal can be approximated as white noise. The autocorrelation function of white noise reaches the greatest value at the zero point, while it is zero at the nonzero points, which is quite different from the autocorrelation function of the general periodic signal. It can be considered that the autocorrelation function energy is mainly concentrated at the zero point for a white noise signal. Therefore, the autocorrelation function is employed to process the acquired signal.

The autocorrelation function reflects the similarity between the signal and itself at different times, which is a statistical method in the time domain, and it can be defined as:

$$
R(\mu)=\int r(t) r(t+\mu) d t
$$

To compute the autocorrelation function of stationary signal $s$ with the length of $N$, firstly, obtain the envelope signal by Hilbert transform of the measured signal $[23,32]$ :

$$
\begin{gathered}
\hat{x}(t)=H\{x(t)\}=\frac{1}{\pi} \int_{-\infty}^{\infty} \frac{x(\tau)}{t-\tau} d \tau \\
y(t)=|\hat{x}(t)|
\end{gathered}
$$

Secondly, remove the DC component:

$$
S t d(y)=\sqrt{\left(\frac{1}{n} \sum_{i=1}^{n}\left(y_{i}-\bar{y}\right)^{2}\right)}
$$




$$
s(t)=\frac{y(t)-\operatorname{mean}(y(t))}{\operatorname{std}(y)}
$$

According to the theory of stochastic process, the one-dimensional auto-correlation of the generalized stationary signal $s$ with the length of $N$ is defined as follows [33]:

$$
R(m)=E\left\{s(n+m) s(n)^{*}\right\}, n=0,1, \ldots \ldots, N-1, m=0, \pm 1, \ldots \ldots, \pm(N-1)
$$

where $E\{$.$\} is the expectation operator and s(n)^{*}$ denotes the conjugation of $s(n)$. As pointed out above, the periodic impulse feature is the most typical sign of a bearing fault. Therefore, we calculated the autocorrelation function of three stimulated signals. Where Signal $1 x(t)$ is the transient impulse fault sequence of rolling bearings, which can be obtained by Equations (14) and (15). The impulse period is set as $T=0.01 \mathrm{~s}$. Signal $2 N(t)$ is the noise signal. Signal 3 is the mixed signal of $x(t)$ and $N(t)$, and the SNR is set to $-8.1 \mathrm{db}$.

$$
\begin{gathered}
x(t)=\sum_{k=-\infty}^{+\infty} h\left(t-K T-\tau_{k}\right) \delta_{j}(i) A_{k} \\
h(t)=\exp \left[\left(-\frac{\varepsilon}{\sqrt{1-2 \varepsilon^{2}}} 2 \pi f_{n} t\right) \sin \left(2 \pi f_{n} t\right)\right] t \geq 0
\end{gathered}
$$

where

$$
\delta_{j}(i)=a\left[\sin \left(2 \pi f_{h} i+\varphi_{j}\right)+c\right] \varphi_{j}=\frac{(j-1) \pi}{2}
$$

The autocorrelation function of three signals can be calculated, as shown in Figure 1a to c, respectively. From Figure 1a, it can be seen that the impulse period of the autocorrelation function for the fault sequence is also $T=0.01 \mathrm{~s}$, which retains the impulse information of the original signal. From Figure 1b, it can be found that the autocorrelation function of a Gaussian white noise signal concentrates its energy at the zero point. In Figure 1c, although the impulse fault feature is submerged by the noise, the impulse period $T=0.01 \mathrm{~s}$ can be easily observed with the calculation of the autocorrelation function. Moreover, the energy of the noise is concentrated at the zero point. Therefore, the impulse fault feature and the noise can be separated through the autocorrelation function.
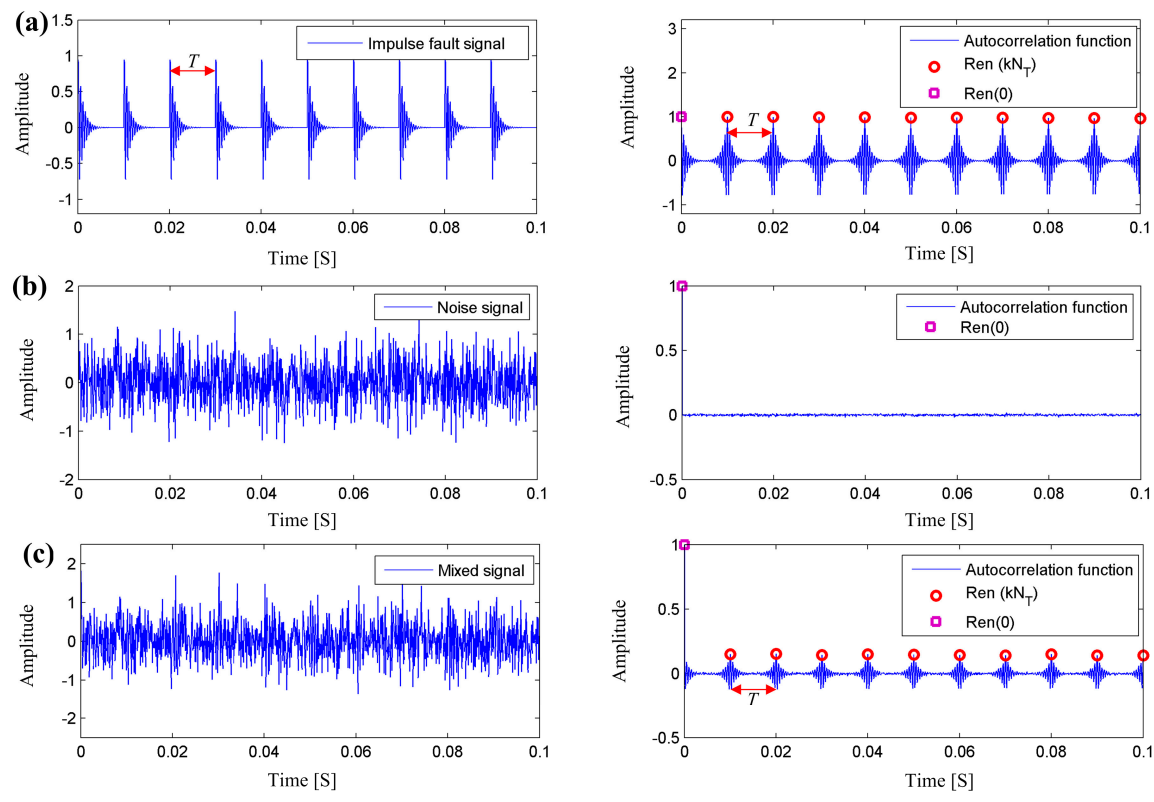

Figure 1. The autocorrelation functions of three kinds of stimulated signals, (a) The autocorrelation function of period impulse fault signal; (b) The autocorrelation function of white noise signal; (c) The autocorrelation function of signal mixed by period impulse fault signal and white noise signal. 
Based on the above analysis, the autocorrelation function impulse harmonic to noise ratio index (ACFHNR) is proposed, as shown in Equation (16):

$$
\mathrm{ACFHNR}=\frac{\sum_{i=1}^{k} R_{e n}\left(k N_{T}\right)}{R_{e n}(0)}
$$

where $\sum_{i=1}^{k} R_{e n}\left(k N_{T}\right)$ is the energy value of the autocorrelation function $R(\mu)$ in the $k$ th period of impulse features. $R_{e n}(0)$ is the energy value of the autocorrelation function $R(\mu)$ at the zero point. It is recommended that $k=3$. Obviously, the larger the ACFHNR index, the more periodic impulse feature information is contained in the vibration signal. The algorithm of the ACFHNR index is shown in Table 1.

Table 1. The algorithm of the autocorrelation function impulse harmonic to noise ratio (ACFHNR) index.

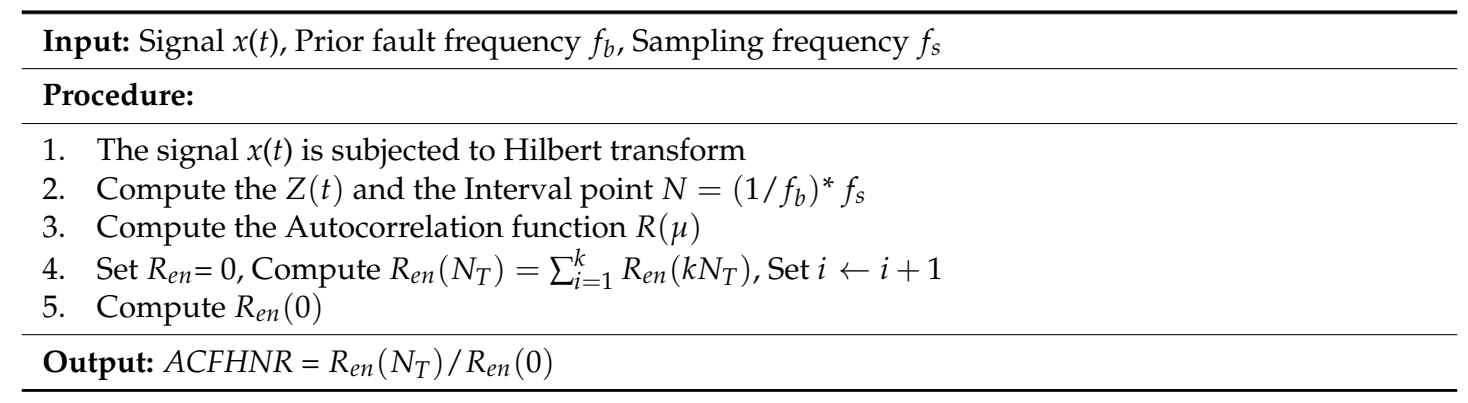

The vibration fault signal of a rolling bearing is sensitive to the kurtosis value. Therefore, we made a comparative analysis of the proposed ACFHNR index and the kurtosis. The kurtosis can be calculated by Equation (17):

$$
\operatorname{Kurt}(i)=\frac{1}{N} \sum_{i=1}^{N}\left(\frac{x(i)-\bar{x}}{\sigma_{t}}\right) 4
$$

where $\bar{x}$ is the mean value of the signal $x(i)$ and $\sigma_{t}$ is the standard deviation of $x(i)$. The tested signal can be obtained by mixing the transient impulse fault sequence $x(t)$ and the noise signal $n(t)$.

$$
S(t)=x(t)+n(t)
$$

Based on the mixed signal $S(t)$, we set the SNR to $-14.5 \mathrm{db}$ to $0 \mathrm{db}$ and calculated the kurtosis value and ACFHNR index. The simulation result is shown in Figure 2.

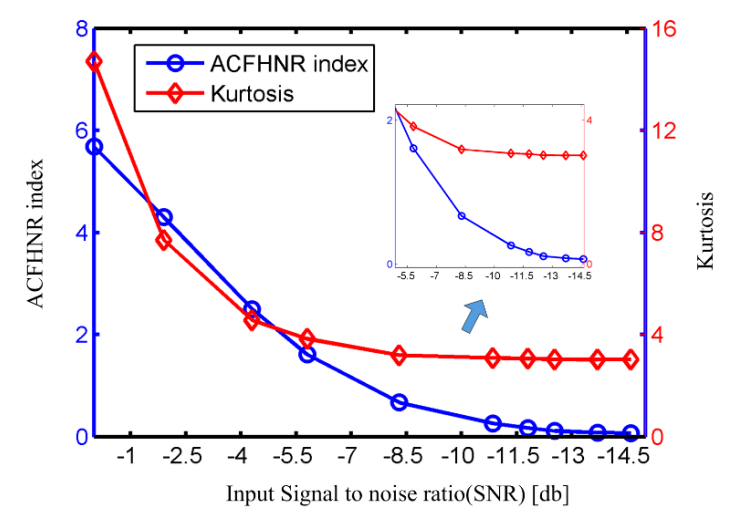

Figure 2. The comparative simulation of the proposed ACFHNR index and the kurtosis. 
From Figure 2, it can be seen that, when the input SNR of the stimulated signal changed from to $0 \mathrm{db}$ to $-14.5 \mathrm{~dB}$, both the kurtosis value and the ACFHNR index gradually decreased, which indicated that both the kurtosis factor and the ACFHNR index can distinguish signals with different SNR of the input signal. However, when the SNR of the input signal is less than $-5.5 \mathrm{db}$ and larger than $-14.5 \mathrm{~dB}$, the ACFHNR index has better resolution than the kurtosis, indicating that the proposed ACFHNR index can be used for identifying the incipient periodic impulse feature of the rolling bearings.

\subsection{Teager Energy Operator}

The Teager energy operator is a nonlinear operator proposed by Teager and Kaiser. It has high temporal resolution and can adaptively detect an instantaneous change of signal [34-36]. As a result, it is suitable for extracting the impulse fault features of rolling bearings. Moreover, as the Teager energy signal contains the total energy of the signal, including the information of amplitude modulation (AM) and frequency modulation (FM) caused by impulse fault feature, the Teager energy operator can improve the signal to noise ratio (SNR) and make the impulse fault feature extracted from a rolling bearing more reliable [34]. It is also capable of increasing the signal-to-noise ratio of the spectrum, sharpening the spectral peaks that reveal the presence of faults.

The Teager energy operator for the continued signal $v(t)$ can be expressed as:

$$
\varphi_{c}[v(t)]=[\dot{v}(t)]^{2}-v(t) \ddot{v}(t)
$$

Moreover, the Teager energy operator for the discrete signal $x(n)$ can be expressed as:

$$
\varphi[x(n)]=[x(n)]^{2}-x(n-1) x(n+1)
$$

Therefore, considering that the Teager energy operator has good adaptability to detect the transient components of a signal, it is employed to detect the fault features of rolling bearings in this paper.

\section{The Proposed Method}

Under the motivation of the above analysis, a method combined the ACFHNR index based on the SVD and Teager energy operator (TEO) demodulation technology is proposed for the early fault diagnosis of rolling bearings. The framework of the proposed method is shown in Figure 3, and its implementation can be described as follows:

Firstly, select the appropriate delay step to construct Hankel matrix. When the computational cost and calculation accuracy are taken into account, there is a trade-off between computational efficiency and decomposition performance when deciding an appropriate choice of $m$ [15]. In this paper, the $m$ is set as $15 \leq m \leq 30$, and the singular value decomposition is performed for the constructed Hankel matrix.

Secondly, for the original vibration signal, the theoretical fault characteristic frequency can be calculated according to the geometry size and defect frequencies multiple of running speeds. Therefore, the prior period of impulses $T$ can be computed through Equation $T=f s / f$, where $f$ is the fault characteristic frequency of the rolling element bearings, while fs is the sampling frequency. With the prior period of impulses $T$, the ACFHNR index for each singular component can be calculated.

Thirdly, the singular component with the largest ACFHNR index is employed to construct the new Hankel matrix. The average of the inverse diagonal elements of the reconstructed Hankel matrix is calculated, which is the denoising signal of the raw vibration signal.

Finally, we implemented the Teager energy operator envelope analysis to the reconstructed signal for early fault diagnosis. The fault type can be decided on the basis of the extracted characteristic frequency information. 


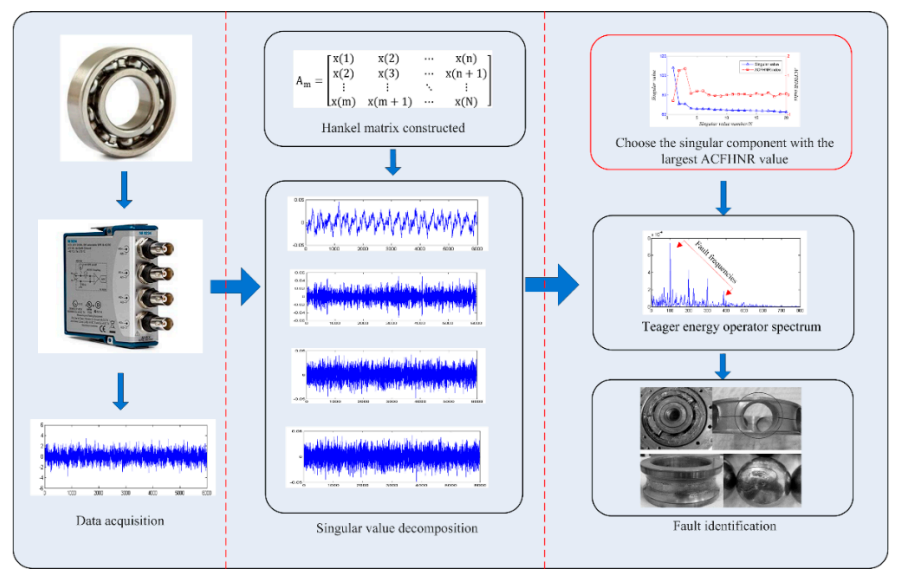

Figure 3. The framework of the proposed method.

\section{Simulation Analysis}

\subsection{The Stimulated Signal}

To verify the validity of the proposed method, the simulated fault signals of the rolling bearing are analyzed in this section. The simulated signal of the early faults of ball bearings can be calculated by Equations (14), (15), (21) and (22), where $I(t)$ is the interference signal, $x(t)$ is the transient impulse fault signal, and $n(t)$ is the noise signal.

$$
\begin{gathered}
S(t)=x(t)+I(t)+n(t) \\
I(t)=A_{h} \sum_{i=1}^{n} \sin \left(2 \pi f_{h i}\right)
\end{gathered}
$$

The simulation result of the fault signal is shown in Figure 4; Figure 4a is the transient periodic impulse fault signal, Figure $4 \mathrm{~b}$ is the interference signal, and Figure $4 \mathrm{c}$ is the noise signal. The impulse period is set to $0.01 \mathrm{~S}$, and the resonance frequency is $2000 \mathrm{HZ}$. Moreover, 6000 sampling points of the signals are obtained. The sampling frequency is set at $12 \mathrm{KHZ}$. The signal-to-noise ratio (SNR) is set to $-13.2 \mathrm{~dB}$, and the signal-to-interference signal ratio (SIR) is set to $-4.5 \mathrm{~dB}$. In order to show the signal clearly, only 3000 sample points are plotted in Figure 4. The mixed signal and its envelope spectrum are plotted in Figure 5. It can be seen that the informative features in the spectrum of the original signal cannot be identified. Therefore, the proposed method is performed on the simulated signal to detect the incipient fault features.
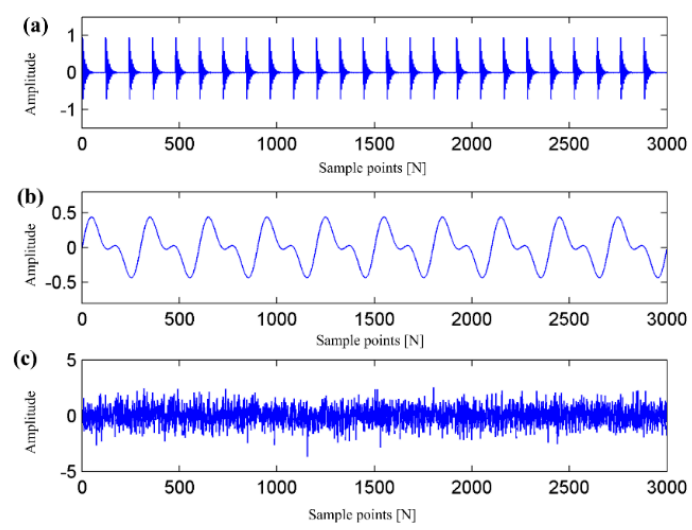

Figure 4. The stimulated fault signals of rolling element bearings. (a) The transient impulse fault signal; (b) The interference signal; (c) The noise signal. 

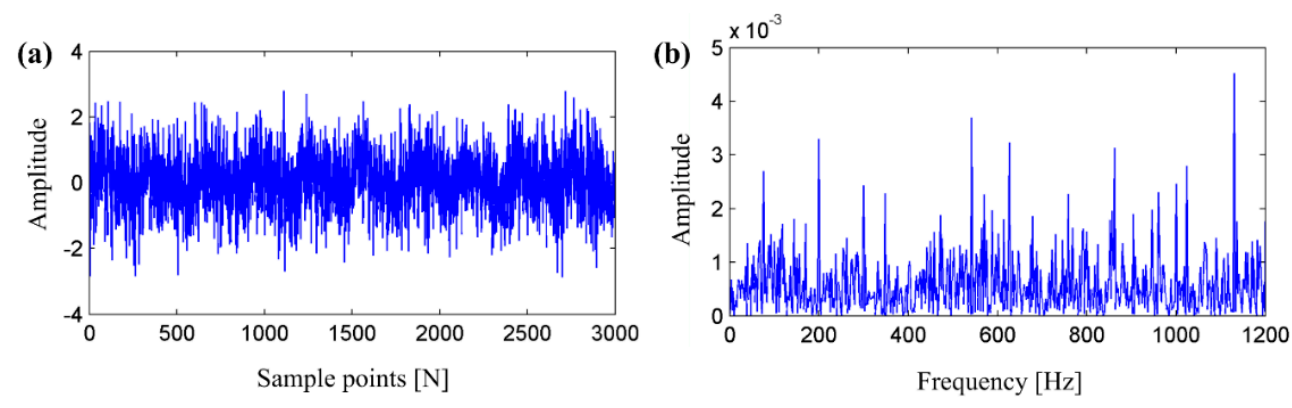

Figure 5. (a) The stimulated fault vibration signal; (b) The envelope spectrum of (a).

\subsection{The Performance Analysis of the Proposed Method}

In order to analyze the decomposition performance of SVD method, we studied the relationship between the singular values of the SVD method and different kinds of signals first. Firstly, the Hankel matrix $\mathbf{A}_{m}$ is constructed when applying the SVD denoise method, and there is a high degree of correlation between the adjacent rows [28]. As a result, the matrix $\mathbf{A}_{m}$ must be a morbid matrix, and its rank $r$ is far less than $l$. Therefore, for an SVD denoising approach, the useful information should be determined by some singular components.

In order to separate the effective periodic impulse component, the interference component, and the noise component of the fault signal, it is important to verify whether there were dislocations filtering the characteristics of the singular value distributions of the different signals. Hence, the periodic impulse fault signal, the interference signal, and the white noise signal are set as reference signals to build three groups of mixed signals. Mixed signal 1 is comprised of the interference signal and the noise signal, and mixed signal 2 is composed of the periodic impulse fault signal and the noise signal, while mixed signal 3 is comprised of the periodic impulse fault signal, the interference signal, and the noise signal. The three reference signals and the three mixed signals are analyzed based on the SVD denoise method. The delay step is set as $m=20$. The singular values of these six signals are obtained, as shown in Figures 6 and 7.

Interestingly, it can be found that the singular values of mixed signals are added by the reference signals, as shown in Figures 6 and 7. For example, the singular values of mixed signal 1 are added by the interference signal and the white noise signal. This showed that different singular values correspond to different kinds of signals. Therefore, the sensitive impulse feature signal can be reconstructed with the appropriate singular components, selected based on certain principles of the SVD method, thereby realizing the fault features extraction of the rolling bearings.

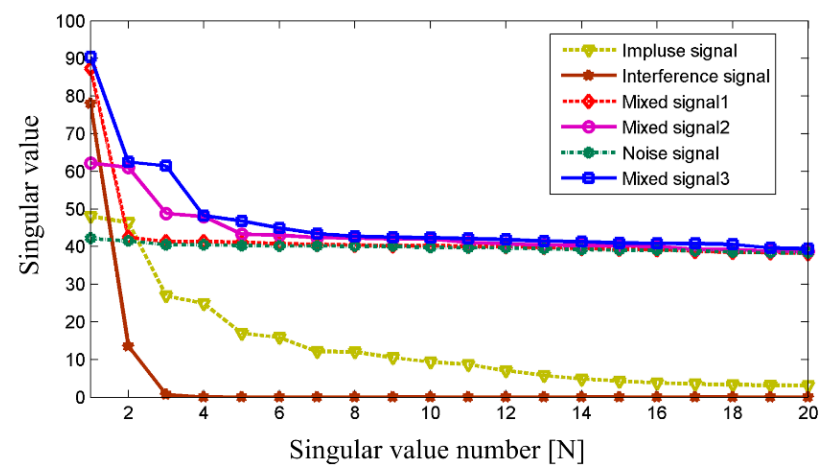

Figure 6. The singular components of these six signals. 
(a)

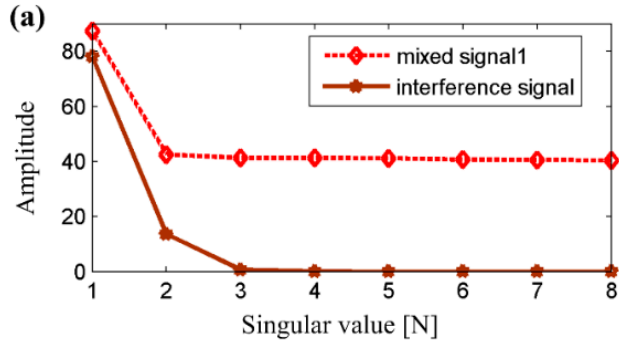

(b)

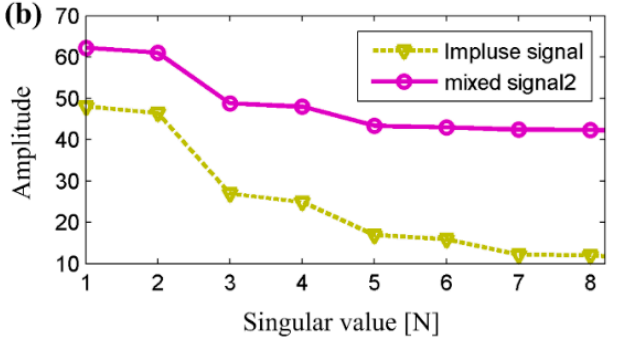

Figure 7. The singular components. (a) The interference signal and mixed signal 1; (b) The impulse signal and mixed signal 2.

In order to verify the performance of proposed ACFHNR-SVD-TEO method, the simulated fault vibration signal of the rolling bearing is analyzed with the feature extraction method. The delay step $m$ is set as $m=20$. For comparison purposes, the kurtosis value and the ACFHNR index value corresponding to the 20 singular components of SVD are computed, as shown in Figure 8. Figure 8 exhibited that the kurtosis and the ACFHNR values of the second and the third singular components are larger than the other values, which tells us that the second and the third singular components are the effective signal features. However, apparently, it can be found that the ACFHNR index is very sensitive to the fault components, as the ACFHNR indexes corresponding to the second and the third singular components are much larger than the others, while the kurtosis did not have such good performance. As a consequence, the ACFHNR index has much better resolution than the kurtosis for extracting fault features.
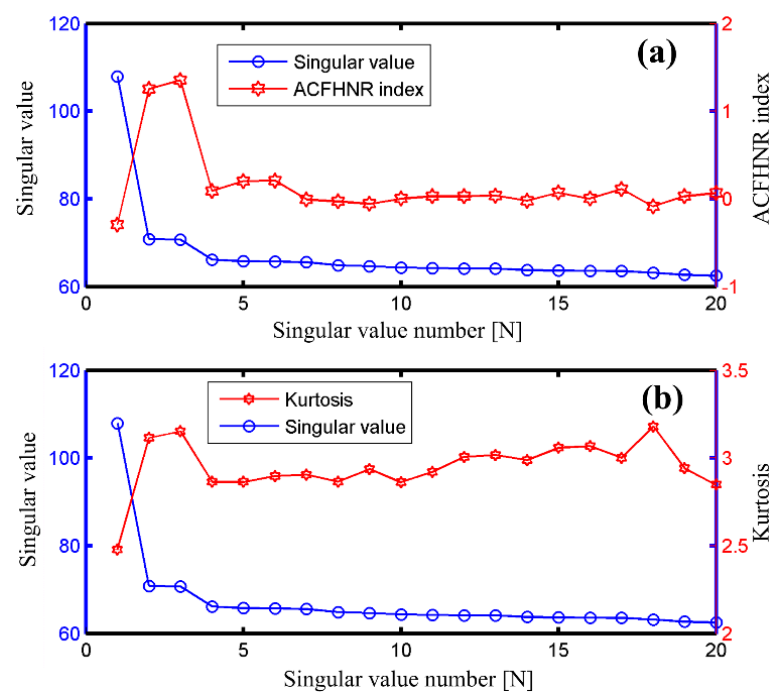

Figure 8. The relationship between the singular value and (a) the kurtosis or (b) the ACFHNR index.

From the analysis above, the decomposition result of the SVD method is shown in Figure 9. Ten singular components are plotted, as shown in Figure 9a. Moreover, the singular components and their Teager energy operator spectra are obtained. Figure $9 b, c$ correspond to the second and the third singular components, respectively. One can easily see that the fault frequency and its harmonics are clearly detected in the second and the third singular components. Based on the above analysis, it can be seen the ACFHNR index is very sensitive to the fault information, which has much better performance than the kurtosis for extracting the weak periodic impulse fault feature. Moreover, the proposed ACFHNR-SVD-TEO method is proved to be effective at extracting the incipient fault features of rolling bearings. 


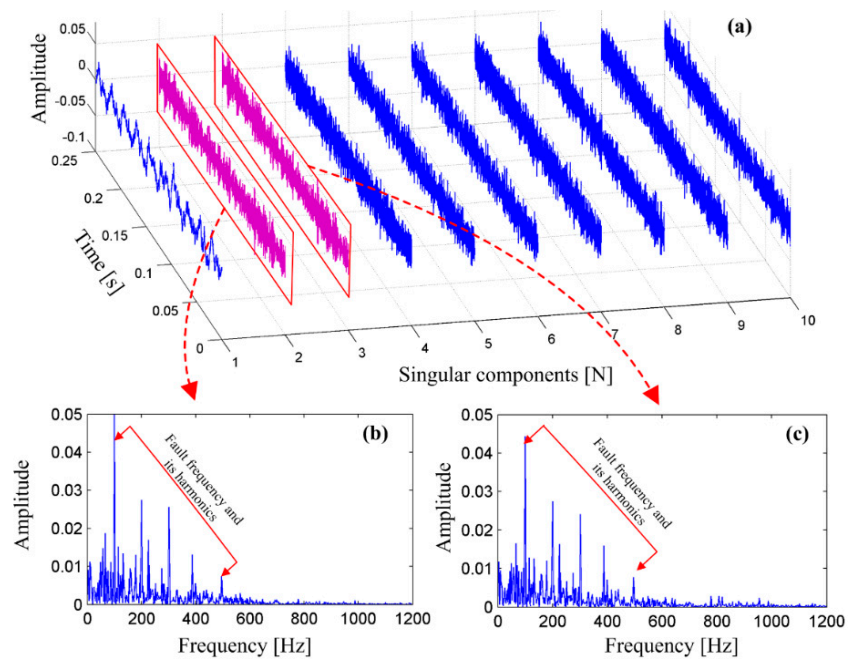

Figure 9. The singular components, (a) their Teager energy operator spectrum; (b) The second spectrum; and (c) The third spectrum.

Additionally, for comparison purposes, other well-established methods such as the median value of singular values based SVD method and the Difference spectrum based SVD method are employed to process the stimulated vibration signal, and the results are shown in Figure 10a,b, respectively. It can be seen that the median value of the singular values based on SVD cannot identify the fault features, as shown in Figure 10a. Although the Difference spectrum based SVD method can detect the fault frequency $f_{b}$ and its second harmonic $2 f_{b}$, the fault features are not obvious, as shown in Figure 10b. The Kurtogram of the stimulated fault vibration signal is shown in Figure 11a, from which the optimal carrier frequency is determined as $2000 \mathrm{HZ}$, and the center frequency is $1000 \mathrm{HZ}$. Therefore, the optimal frequency band is $1000 \mathrm{HZ}$ to $3000 \mathrm{HZ}$. As a result, the band-pass filter is designed to extract the potential effective fault features from the vibration signal. The envelop spectrum of the filtered signal is presented in Figure 11b. It can be found that the fault frequency $3 f_{b}$ and $4 f_{b}$ can be detected. However, the fault features are also not obvious. Therefore, traditional well-established SVD denoising and the fast Kurtogram can obtain little diagnostic information.
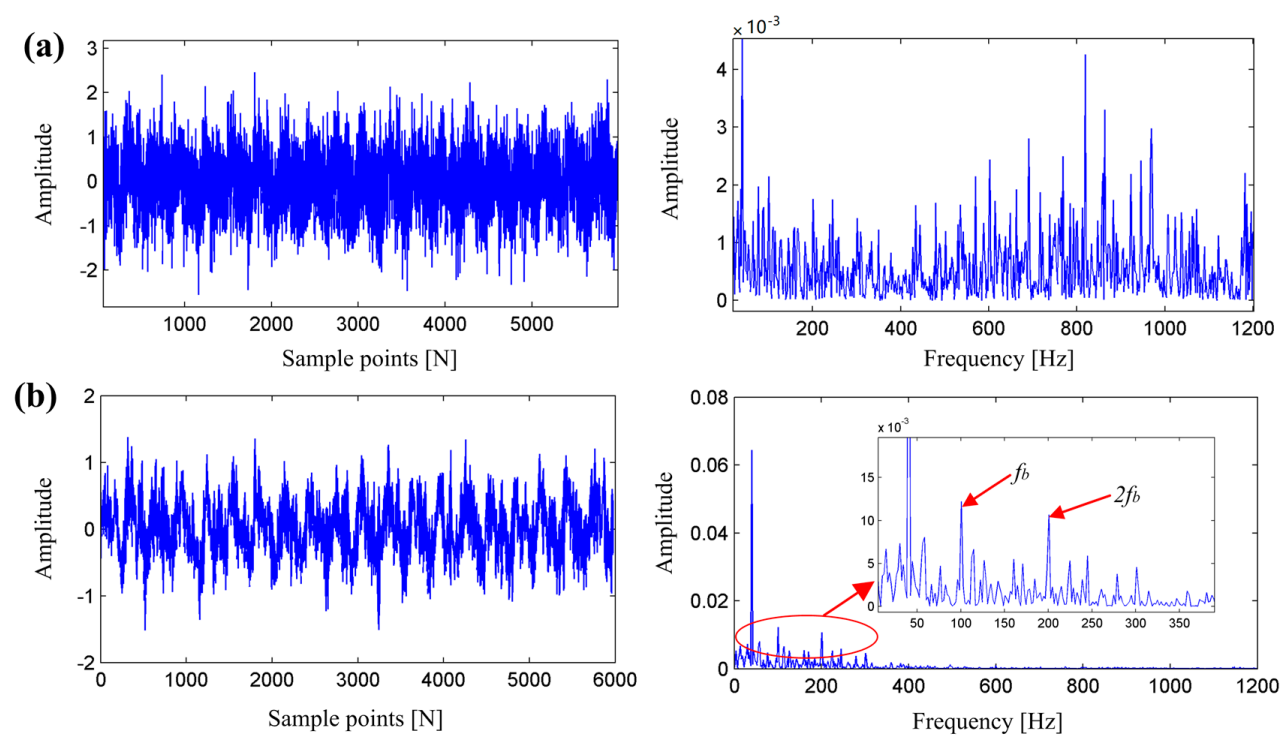

Figure 10. The extracted feature signals and their envelop spectrum. (a) The median value of singular values based SVD method; (b) The Difference spectrum singular values based SVD method. 
(a)

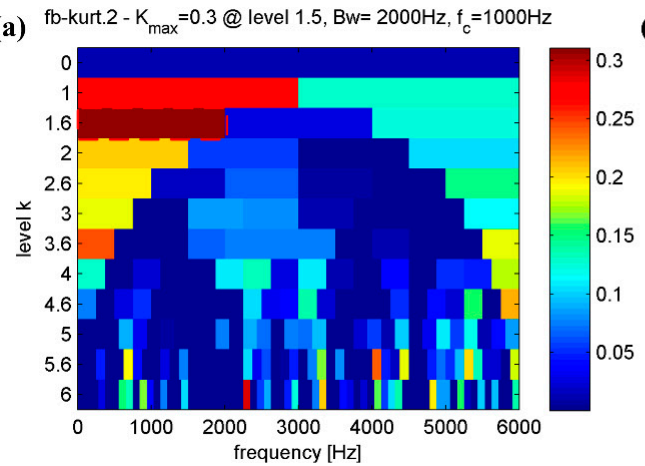

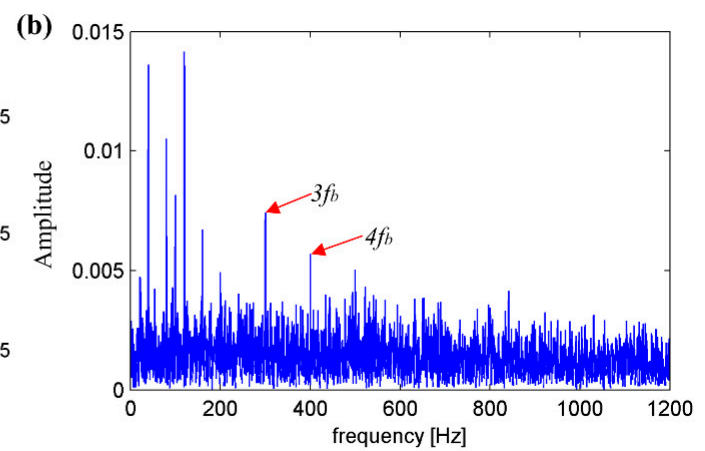

Figure 11. (a) Optimal resonance band obtained by Kurtogram; (b) envelope spectrum.

\section{Experiment Results}

In this section, the proposed method is verified by the experimental data. The fault data for the bearing roller comes from the bearing data center at the Laboratory of Case Western Reserve University. The test bed consists of two Horsepower (HP) motors, a torque transducer, and a dynamometer, as shown in Figure 12. The test bearings (SKF6205, Svenska Kullager-Fabriken group. Switzerland), including the drive end and fan end bearings, support the motor rotor shaft. Three types of Single point faults (outer race fault (ORF), inner race fault (IRF), and ball fault (BF) were introduced to the test bearings using electro-discharge machining [37]. All bearing fault data are annotated with bearing geometric, operating condition, and fault information. In the experiment, the vibration signals of the rolling bearing were collected by three accelerometers, which were mounted to the housing with magnetic bases at the three, six, and 12 o'clock positions. The fault frequency of the ball fault can be computed as follows:

$$
f_{b p f r}=\frac{D}{2 d}\left(1-\left(\frac{d}{D}\right)^{2} \cos ^{2} \alpha\right) f_{r}
$$

where $f_{r}$ is the rotating frequency, $D$ is the pitch diameter, $d$ is the balling diameter, and $\alpha$ is the contact angle. According to the geometry size and defect frequencies multiple of running speed, the fault frequency of the ball fault is obtained, which is about $f_{b p f r}=137 \mathrm{~Hz}$.

In this section, the vibration signals of the drive end bearing are analyzed. To verify the effectiveness of the proposed method, ball fault 1 , with fault diameters of seven miles, is analyzed. The rotation speed of ball fault 1 is $1772 \mathrm{r} / \mathrm{min}$. The sampling frequency is set to $12 \mathrm{kHz}$ in the experiment. The original signal and its envelope spectrum are plotted in Figure 13. From Figure 13, it can be found that fault frequencies are flooded by noise and irrelevant harmonic components. Therefore, the proposed ACFHNR-SVD-TEO method is employed for incipient fault feature extraction of the fault signal.

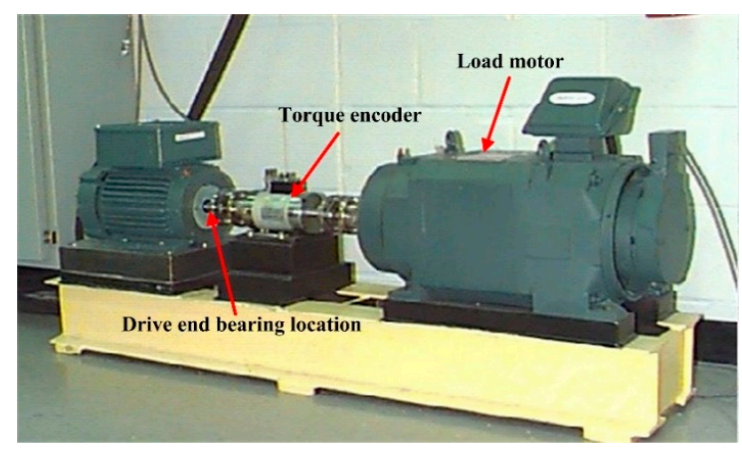

Figure 12. The bearing fault test bed from the bearing data center at the Laboratory of Case Western Reserve University. 

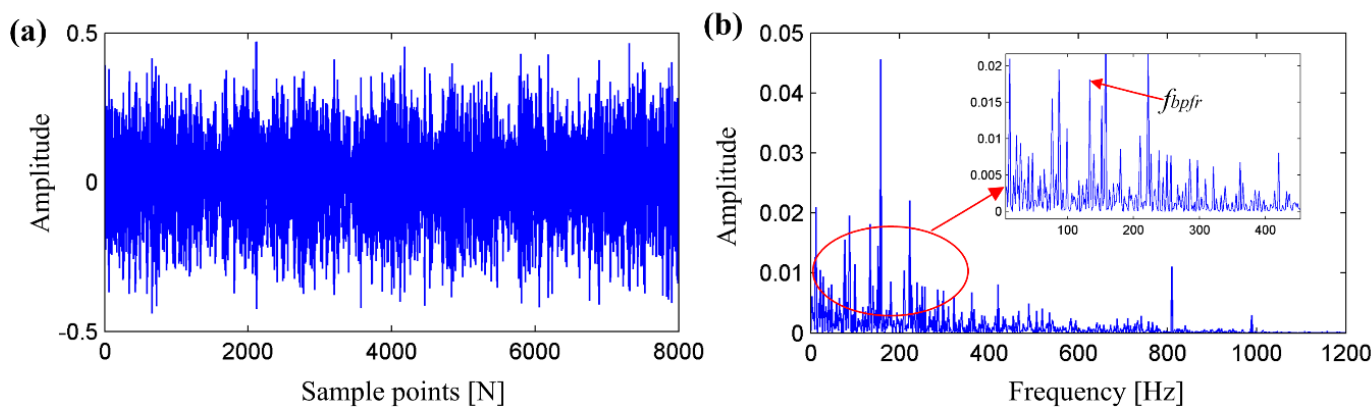

Figure 13. The original signal and the envelope spectrum of ball fault 1.(a) The raw fault signal ; (b) The envelope spectrum of the raw fault signal.

Firstly, the delay step is set as $m=15$. The kurtosis value and the ACFHNR index value corresponding to these 15 singular components of SVD are calculated, as shown in Figure 14. Figure 14 exhibited that the ACFHNR value of the first singular component is larger than the ACRHNR values of the other components, which tells us that the first component is the effective signal feature. Therefore, the first singular component is processed, and its Teager energy operator spectrum is

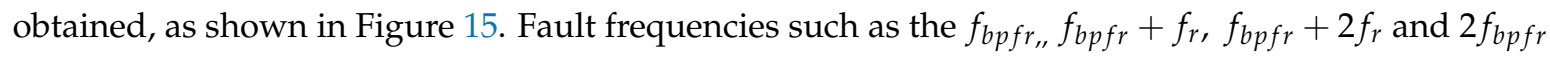
can be clearly identified, demonstrating that the proposed method can successfully detect the incipient fault features of rolling element bearings. Also, it shows that the first singular component is the effective component. However, the kurtosis values corresponding to the third and fourth singular components are much larger than the other kurtosis values, as shown in Figure 14, indicating that the kurtosis values may provide the wrong information for extracting the sensitive fault information.

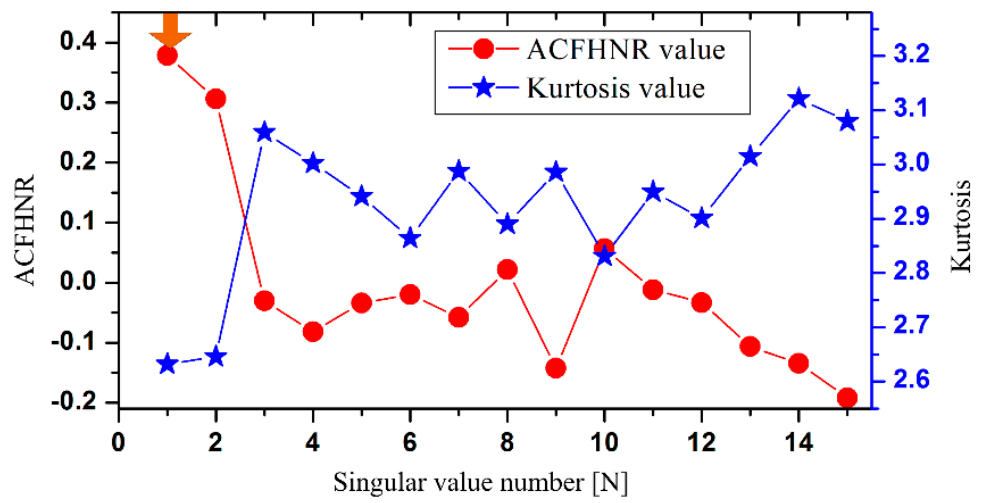

Figure 14. The comparison of the proposed ACFHNR index and the kurtosis.
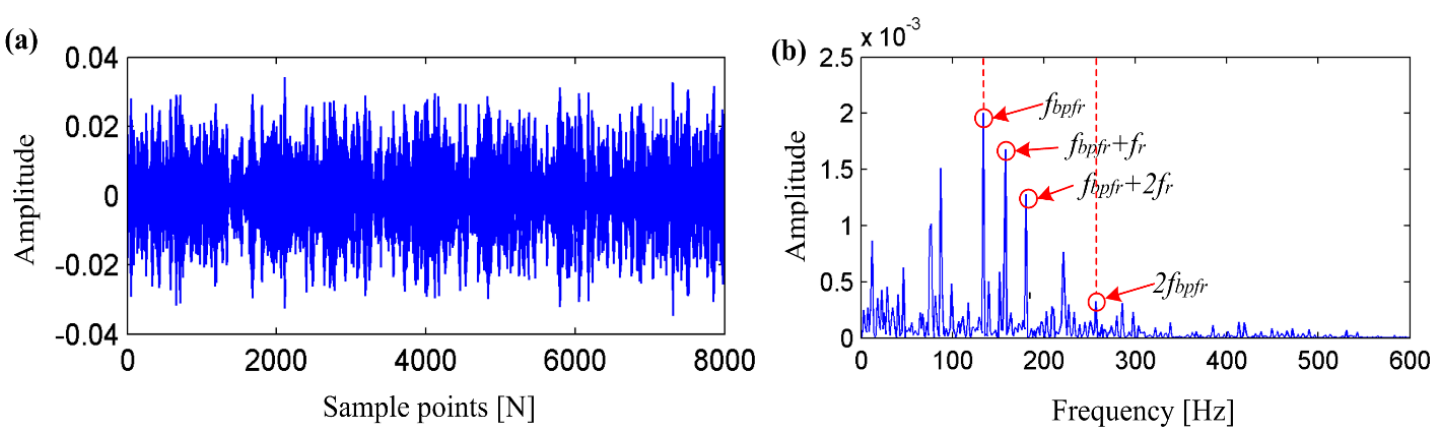

Figure 15. (a) The extracted fault feature signal and the (b) Teager energy operator spectrum of ball fault 1 . 
To further evaluate the performance of the proposed method, the fault data for a bearing roller from the Intelligent Maintenance System, University of Cincinnati, are explored. In the test bed, two PCB 353B33 High Sensitivity Quartz ICP (Integrated Circuits Piezoelectric) accelerometers (PCB Piezotronics Inc., Depew, NY, USA) are mounted for data acquisition (one vertical and one horizontal) for each bearing, and a radial load of $2721.6 \mathrm{~kg}$ is applied to the bearings. Meanwhile, the vibration signals are measured at an interval of every $10 \mathrm{~min}$. The sampling frequency is set to $20 \mathrm{KHz}$, and the data length is 20,480 points [38]. The shaft rotating speed of the motor is $2000 \mathrm{rpm}$. Four bearings, named Bearings 1 to 4, are installed on one shaft, as shown in Figure 16. At the end of the accelerated experiment, an outer race fault is discovered in test bearing 1 .
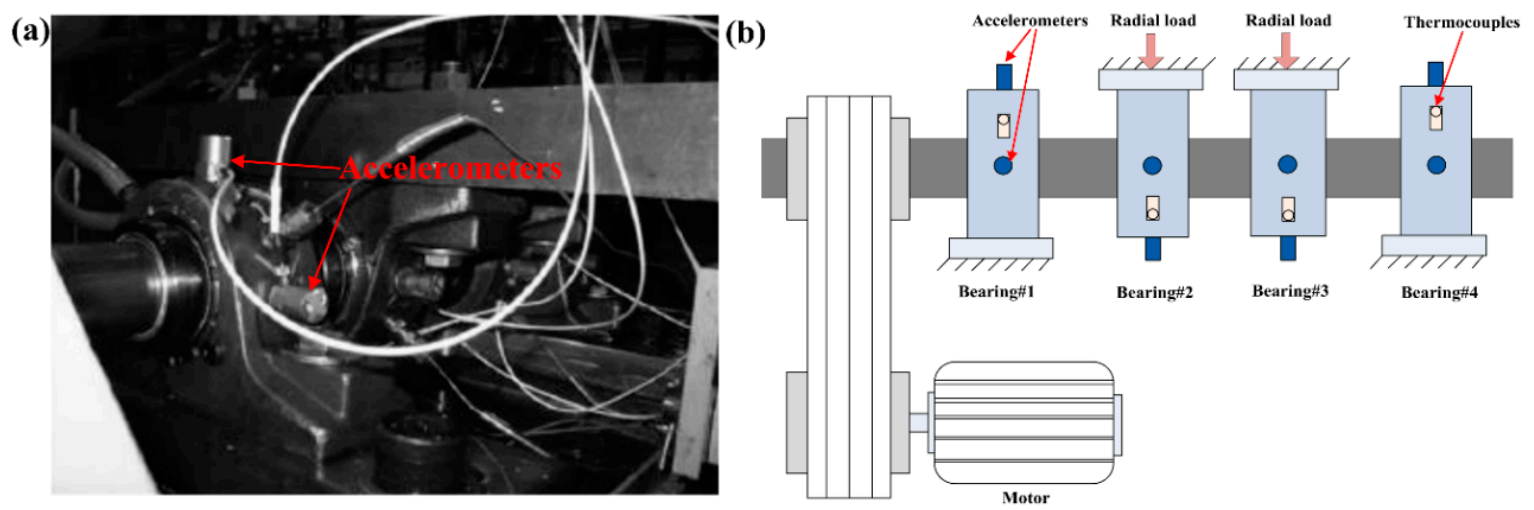

Figure 16. The bearing fault test bed from the Intelligent Maintenance System, University of Cincinnati, (a) The physical map; (b) The sketch map.

The fault frequency of the outer race fault can be computed as follows:

$$
f_{b p f o}=\frac{1}{2} Z\left(1-\frac{d}{D} \cos \alpha\right) f_{r}
$$

where $f_{r}$ is the rotating frequency, $\mathrm{D}$ is the pitch diameter, $\mathrm{d}$ is the balling diameter, $\mathrm{Z}$ is the number of the balls, and $\alpha$ is the contact angle. Also, according to Equation (23), the fault frequency of the out racer of the bearing can be computed, which is about $236 \mathrm{HZ}$ [29]. Based on this prior fault frequency, the ACFHNR index value of all the fault vibration signals in the whole life of the testing are calculated, as shown in Figure 17a. For comparison purposes, the kurtosis and RMS (Root Mean Square) values are computed. The results are shown in Figures 18a and 19a, in which the sub graphs in the figures are the local enlargement of dashed line boxes.

In the run-to-failure experiment, it is assumed that the bearing worked at the stationary stage from group 10 to group 500. The discrete probability distribution for the ACFHNR, kurtosis, and RMS index at the stationary stage is Gaussian distribution, as presented in Figure 20. The alarm thresholds for each parameter are set as the mean value plus four times the stand deviation [23]. Then the threshold of the three parameters can be obtained, as shown in Table 2. Also, the alarm time for RMS, kurtosis and the ACFHNR index are derived. The alarm time of RMS is $88 \mathrm{~h} 40 \mathrm{~min}$, while that of kurtosis is $107 \mathrm{~h} 50 \mathrm{~min}$, and the ACFHNR warn time is $88 \mathrm{~h} 40 \mathrm{~min}$, as shown in Figures 17a, 18a and 19a. Meanwhile, compared with the envelope harmonic-to-noise ratio (EHNR) index proposed in the literature [23], the ACFHNR index can detect faults $1 \mathrm{~h}$ and $50 \mathrm{~min}$ earlier, where the alarm time of the EHNR index is $90 \mathrm{~h} 30 \mathrm{~min}$ [23].

Moreover, we proposed the concept of sensitivity to quantify the three indexes, which can be calculated by Equation (25), where $\Delta d$ is the difference value of the three indexes in the time $\Delta t$. The sensitivity can be deemed the gradient of the fitted curve in the incipient fault stage. 


$$
S=\frac{\Delta d}{\Delta t}
$$

The sensitivity values in the incipient stages of the three indexes are calculated. For the ACFHNR and RMS indexes, the data from group 532 to group 700 are calculated, as shown in Figures 17b and 18b. For the kurtosis index, the data from group 647 to group 700 are calculated, as shown in Figure 19b. The sensitivity values for the three indexes are presented in Table 3, and it can be found that the sensitivity value of ACFHNR is much larger than that of kurtosis or RMS.
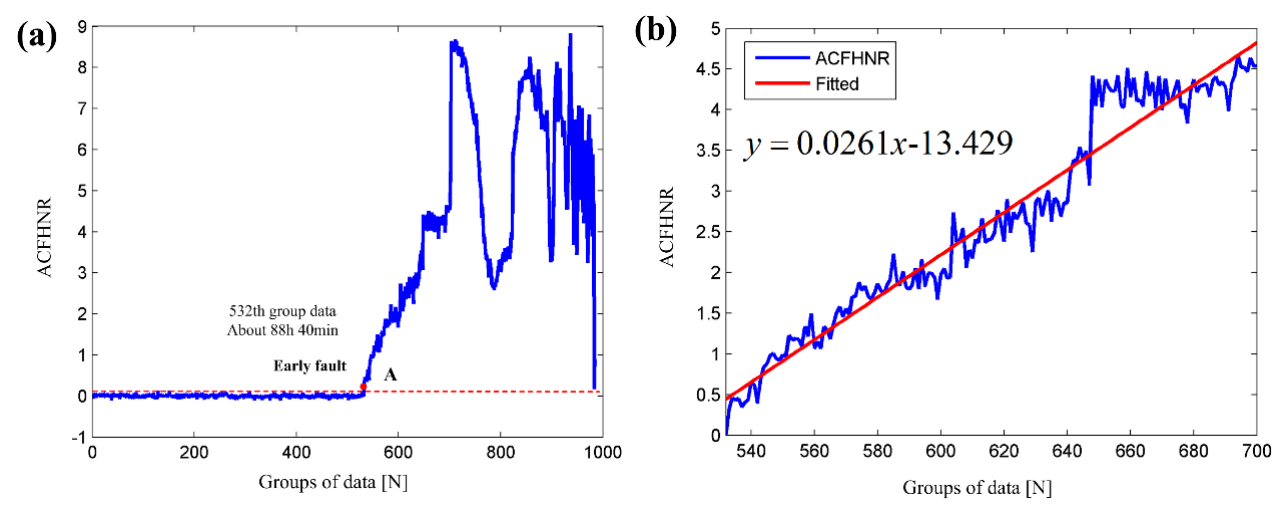

Figure 17. (a) The ACFHNR value for the full life cycle of bearing 1; (b) The sensitivity value of ACFHNR.
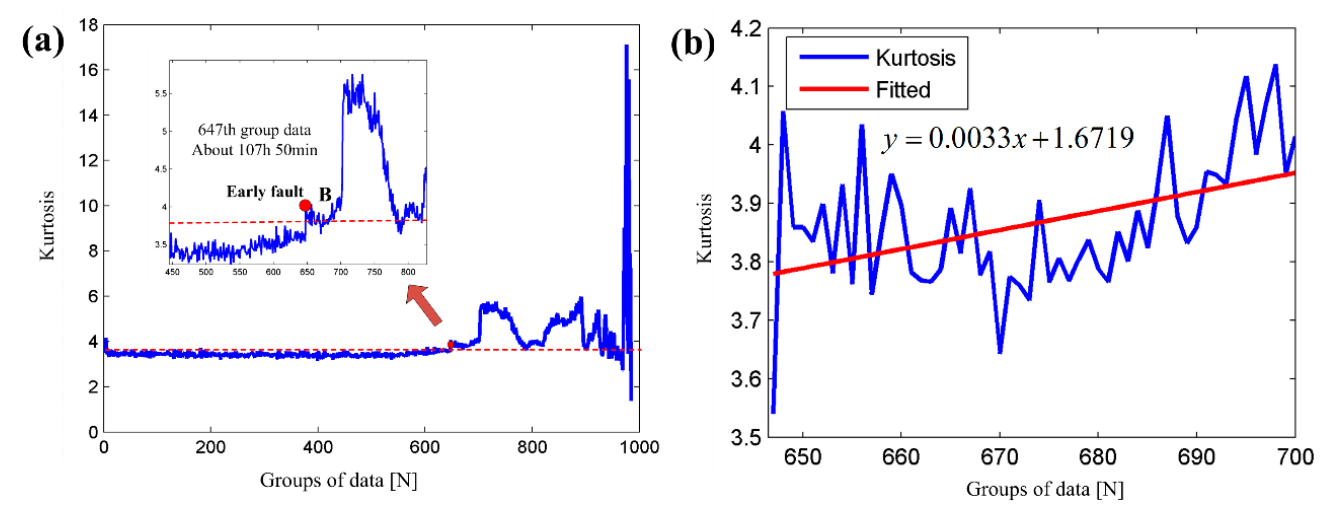

Figure 18. (a) The kurtosis value for the full life cycle of bearing 1 ; (b) The sensitivity value of kurtosis.
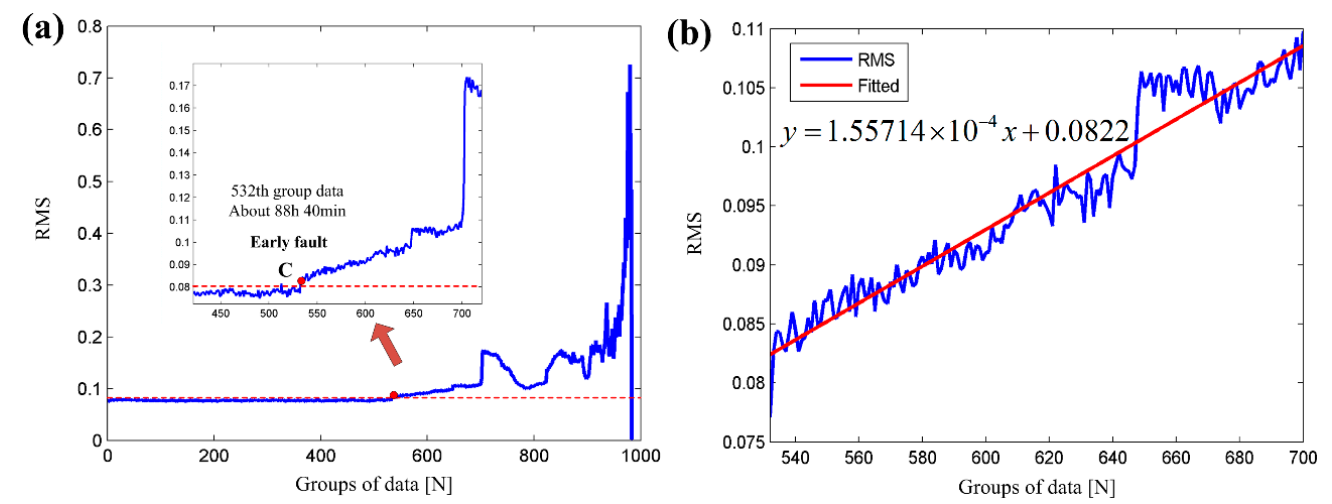

Figure 19. (a) The RMS value for the full life cycle of bearing 1; (b) The sensitivity value of RMS. 

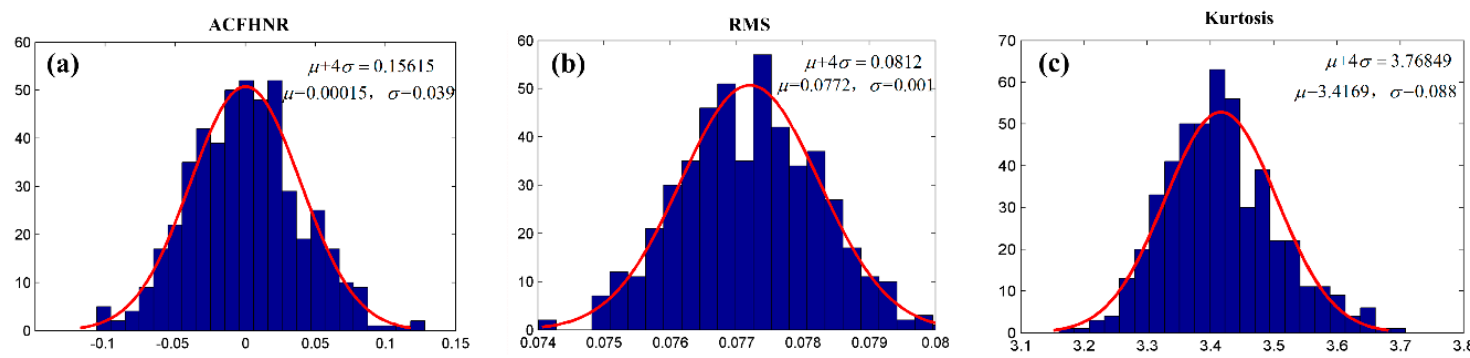

Figure 20. The discrete probability distribution for parameters at the stationary stage: (a) ACFHNR; (b) root-mean-square (RMS); (c) kurtosis.

Table 2. The threshold values of the three indexes.

\begin{tabular}{cccc}
\hline The Index & ACFHNR & Kurtosis & RMS \\
\hline Threshold value & 0.15615 & 3.76849 & 0.0812 \\
\hline
\end{tabular}

Table 3. The sensitivity value of the three indexes in the incipient fault stage.

\begin{tabular}{cccc}
\hline The Index & ACFHNR & Kurtosis & RMS \\
\hline Sensitivity & 0.0261 & 0.0033 & $1.55714 \times 10^{-4}$ \\
\hline
\end{tabular}

From Figures 17-20, at least two conclusions can be drawn: (1) Both the RMS and the ACFHNR index are able to detect faults earlier than the kurtosis factor, as they can detect the fault information at group 532 (at a time of about $88 \mathrm{~h} 40 \mathrm{~min}$ ) while the kurtosis factor detected the fault information at group 647 (at time of about $107 \mathrm{~h} 50 \mathrm{~min}$ ); (2) Compared with the kurtosis and RMS indexes, the sensitivity value of the ACFHNR index is the largest in the incipient fault stage, where the sensitivity value of ACFHNR index is 0.0261 , while the sensitivity values of kurtosis and the RMS are 0.0033 and $1.55714 \times 10^{-4}$, respectively. According to the above conclusions, it can be found that the ACFHNR index is superior to the traditional RMS and kurtosis indexes for detecting the early faults of rolling bearings.

Moreover, the early fault vibration signal of group 533 is analyzed. The sample lengths are set as 10,000. The original vibration signal and its corresponding envelope demodulation spectrum are shown in Figure 21. Theouter race fault frequency $f_{b p f o}=236 \mathrm{HZ}$ can be detected, which further indicates the effectiveness of the ACFHNR index for detecting the early weak fault vibration signal directly. To further prove the usefulness of the proposed ACFHNR-SVD-TEO method, the vibration signal of group 533 is processed.

(a)

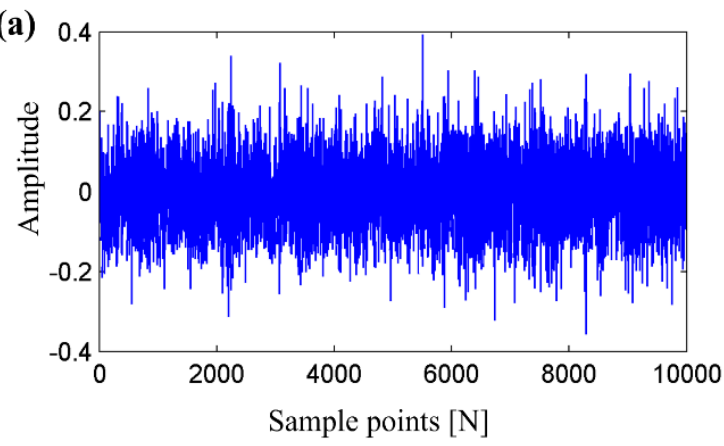

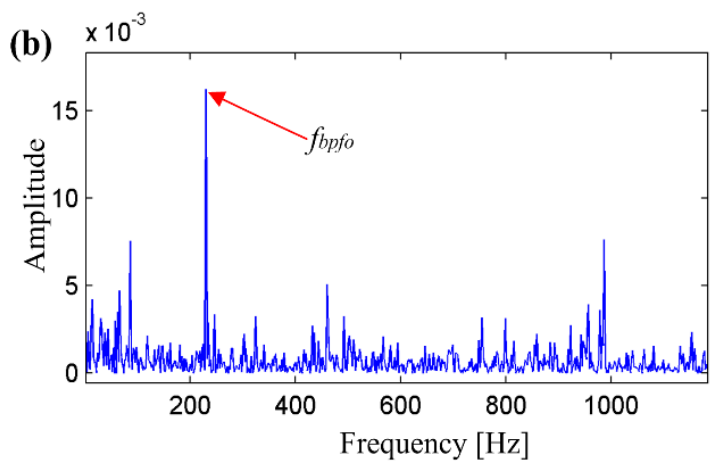

Figure 21. (a)The original vibration signal of group 533; (b) The envelope demodulation spectrum of group 533. 
The delay step is set as $m=15$. Also, both the kurtosis values and the ACFHNR index values corresponding to these 15 singular components are calculated, as shown in Figure 22. It can be seen that the ACFHNR value of the first singular component is the largest, which means that the first component is the effective signal feature. Thus the signal of the first singular component is processed using the proposed method, as shown in Figure 23. The fault frequencies $f_{b p f_{o}}, 2 f_{b p f_{o}}, 3 f_{b p f_{o}}$, and $4 f_{b p f_{o}}$ can be clearly detected. Once again, this processed result proves the usefulness of the proposed method to detect the early faults of the rolling element bearings.

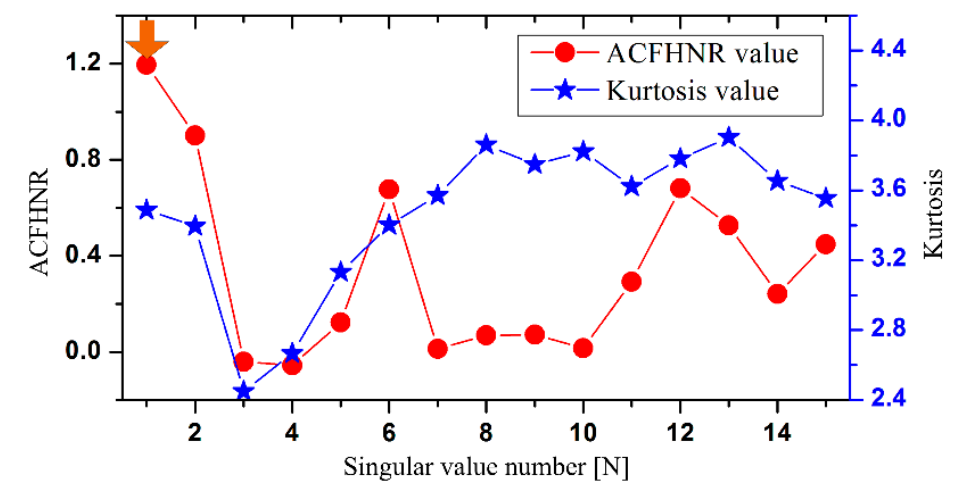

Figure 22. The comparison of the proposed ACFHNR index and the kurtosis.

(a)

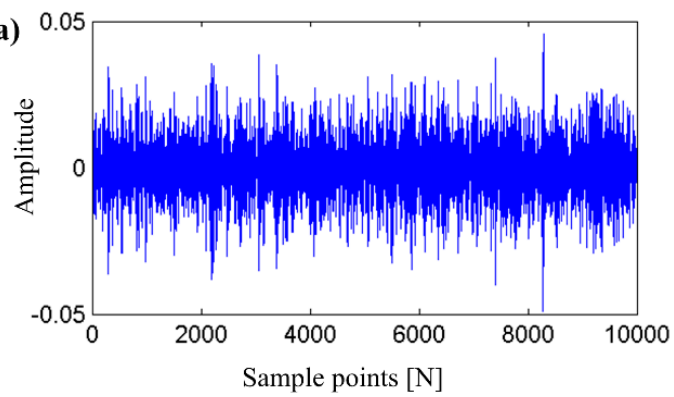

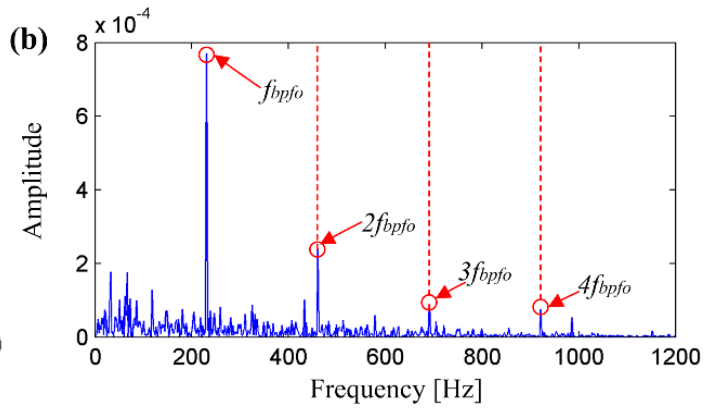

Figure 23. (a)The extracted fault feature signal of of group 533; (b) The teager energy operator spectrum of group 533.

\section{Conclusions}

The incipient periodic impulse feature of the vibration signal from fault rolling element bearings is often contaminated by noise and interference harmonics. In this paper, a novel early fault detection approach based on ACFHNR-SVD combined with Teager energy operator is presented for extracting the incipient periodic impulse feature. The main contributions of this paper can be summarized as follows:

(1) Compared with the kurtosis index, the sensitive singular components obtained by the SVD method can be clearly identified with the proposed ACFHNR index. Moreover, the incipient fault features of the vibration signal from the rolling element bearings can be detected by the proposed ACFHNR-SVD method combined with Teager energy operator method. They are proved by the simulation and experimental results.

(2) The ACFHNR index is able to detect faults earlier than the kurtosis index. Moreover, compared with kurtosis and RMS indexes, the sensitivity value of the ACFHNR index is the largest in the incipient fault stage, demonstrating its superiority over the traditional RMS and kurtosis indexes for detecting the early fault features of the vibration signal. This is proved by the full life cycle of the bearing degradation data. 
In this preliminary study, the ACFHNR index and the proposed ACFHNR-SVD-TEO method are tested and demonstrated to be effective in bearing fault detection using the simulation signal and two sets of experimental data. In our future work, the applicability of the ACFHNR index will be considered for other methods such as ensemble empirical mode decomposition and local mean decomposition.

Acknowledgments: This research was supported by the Chongqing Research Program of Basic Research and Frontier Technology (Grant No. cstc2017jcyjAX0151), the Science and Technology Research Program of the Chongqing Municipal Education Commission (Grant No. KJ1704109), and the National Natural Science Foundation of China (Grant No. 51775076). The authors would like to thank the Case Western University Bearing Data Center and Intelligent Maintenance System, University of Cincinnati, for providing the experimental data.

Author Contributions: Kai Zheng wrote the manuscript and analyzed the data. Tianliang Li and Xiangyu Zhou analyzed and interpreted the data. After the manuscript was finished, Bin Zhang, Yi Zhang and Jiufei Luo reviewed the manuscript.

Conflicts of Interest: The authors declare no conflict of interest.

\section{References}

1. Qiao, Z.; Pan, Z. SVD principle analysis and fault diagnosis for bearings based on the correlation coefficient. Meas. Sci. Technol. 2015, 26, 085014. [CrossRef]

2. Li, H.; Lian, X.; Guo, C.; Zhao, P. Investigation on early fault classification for rolling element bearing based on the optimal frequency band determination. J. Intell. Manuf. 2015, 26, 189-198. [CrossRef]

3. Lei, Y.; Lin, J.; He, Z.; Zuo, M.J. A review on empirical mode decomposition in fault diagnosis of rotating machinery. Mech. Syst. Signal Process. 2013, 35, 108-126. [CrossRef]

4. Aydin, I.; Karakose, M.; Akin, E. Combined intelligent methods based on wireless sensor networks for condition monitoring and fault diagnosis. J. Intell. Manuf. 2015, 26, 717-729. [CrossRef]

5. Golafshan, R.; Sanliturk, K.Y. SVD and Hankel matrix based de-noising approach for ball bearing fault detection and its assessment using artificial faults. Mech. Syst. Signal Process. 2016, 70, 36-50. [CrossRef]

6. Wang, J.; Qiao, L.; Ye, Y.; Chen, Y.Q. Fractional envelope analysis for rolling element bearing weak fault feature extraction. IEEE/CAA J. Autom. Sin. 2017, 4, 353-360. [CrossRef]

7. Georgoulas, G.; Loutas, T.; Stylios, C.D.; Kostopoulos, V. Bearing fault detection based on hybrid ensemble detector and empirical mode decomposition. Mech. Syst. Signal Process. 2013, 41, 510-525. [CrossRef]

8. Zhao, M.; Lin, J.; Xu, X.; Lei, Y. Tacholess envelope order analysis and its application to fault detection of rolling element bearings with varying speeds. Sensors 2013, 13, 10856-10875. [CrossRef] [PubMed]

9. Chandra, N.H.; Sekhar, A.S. Fault detection in rotor bearing systems using time frequency techniques. Mech. Syst. Signal Process. 2016, 72-73, 105-133. [CrossRef]

10. Zhang, Z.; Wang, Y.; Wang, K. Fault diagnosis and prognosis using wavelet packet decomposition, Fourier transform and artificial neural network. J. Intell. Manuf. 2013, 24, 1213-1227. [CrossRef]

11. Jin, S.; Lee, S.K. Bearing fault detection utilizing group delay and the Hilbert-Huang transform. J. Mech. Sci. Technol. 2017, 31, 1089-1096. [CrossRef]

12. Zheng, K.; Zhang, Y.; Zhao, C.; Li, T. Fault Diagnosis for Supporting Rollers of the Rotary Kiln Using the Dynamic Model and Empirical Mode Decomposition. Mechanics 2016, 22, 198-205. [CrossRef]

13. Singh, J.; Darpe, A.K.; Singh, S.P. Bearing damage assessment using Jensen-Rényi Divergence based on EEMD. Mech. Syst. Signal Process. 2017, 87, 307-339. [CrossRef]

14. Xu, T.; Yin, Z.; Cai, D.; Zheng, D. Fault diagnosis for rotating machinery based on Local Mean Decomposition morphology filtering and Least Square Support Vector Machine. J. Intell. Fuzzy Syst. 2017, 32, 2061-2070. [CrossRef]

15. Zhao, M.; Jia, X. A novel strategy for signal denoising using reweighted SVD and its applications to weak fault feature enhancement of rotating machinery. Mech. Syst. Signal Process. 2017, 94, 129-147. [CrossRef]

16. Jiang, H.; Chen, J.; Dong, G.; Liu, T.; Chen, G. Study on Hankel matrix-based SVD and its application in rolling element bearing fault diagnosis. Mech. Syst. Signal Process. 2015, 52, 338-359. [CrossRef]

17. Muruganatham, B.; Sanjith, M.A.; Krishnakumar, B.; Murty, S.S. Roller element bearing fault diagnosis using singular spectrum analysis. Mech. Syst. Signal Process. 2013, 35, 150-166. [CrossRef]

18. Hernandez-Vargas, M.; Cabal-Yepez, E.; Garcia-Perez, A. Real-time SVD-based detection of multiple combined faults in induction motors. Comput. Electr. Eng. 2014, 40, 2193-2203. [CrossRef] 
19. Cong, F.; Zhong, W.; Tong, S.; Tang, N.; Chen, J. Research of singular value decomposition based on slip matrix for rolling bearing fault diagnosis. J. Sound Vib. 2015, 344, 447-463. [CrossRef]

20. Zhao, X.; Ye, B. Selection of effective singular values using difference spectrum and its application to fault diagnosis of headstock. Mech. Syst. Signal Process. 2011, 25, 1617-1631. [CrossRef]

21. Han, T.; Jiang, D.; Wang, N. The fault feature extraction of rolling bearing based on EMD and difference spectrum of singular value. Shock Vib. 2016, 2016, 5957179. [CrossRef]

22. Wang, H.; Chen, J.; Dong, G. Feature extraction of rolling bearing's early weak fault based on EEMD and tunable Q-factor wavelet transform. Mech. Syst. Signal Process 2014, 48, 103-119. [CrossRef]

23. Xu, X.; Zhao, M.; Lin, J.; Lei, Y. Envelope harmonic-to-noise ratio for periodic impulses detection and its application to bearing diagnosis. Measurement 2016, 91, 385-397. [CrossRef]

24. Jiang, Y.; Tang, B.; Qin, Y.; Liu, W. Feature extraction method of wind turbine based on adaptive Morlet wavelet and SVD. Renew. Energy 2011, 36, 2146-2153. [CrossRef]

25. Wang, Z.; Lu, C.; Wang, Z.; Liu, H.; Fan, H. Fault diagnosis and health assessment for bearings using the Mahalanobis-Taguchi system based on EMD-SVD. Trans. Inst. Meas. Control 2013, 35, 798-807. [CrossRef]

26. Liu, Y.; Yu, Z.; Zeng, M.; Zhang, Y. LLE for submersible plunger pump fault diagnosis via joint wavelet and SVD approach. Neurocomputing 2016, 185, 202-211. [CrossRef]

27. Xu, J.; Tong, S.; Cong, F.; Chen, J. Slip Hankel matrix series-based singular value decomposition and its application for fault feature extraction. IET Sci. Meas. Technol. 2017, 11, 464-472. [CrossRef]

28. Ding, J. Detection of the dynamic imbalance with cardan shaft in high-speed train applying eemd-hankel-svd. J. Mech. Eng. 2015, 51, 143. [CrossRef]

29. Li, Y.; Liang, X.; Xu, M.; Huang, W. Early fault feature extraction of rolling bearing based on ICD and tunable Q-factor wavelet transform. Mech. Syst. Signal Process. 2017, 86, 204-223. [CrossRef]

30. Zhao, S.; Liang, L.; Xu, G.; Wang, J.; Zhang, W. Quantitative diagnosis of a spall-like fault of a rolling element bearing by empirical mode decomposition and the approximate entropy method. Mech. Syst. Signal Process 2013, 40, 154-177. [CrossRef]

31. Imaouchen, Y.; Kedadouche, M.; Alkama, R.; Thomas, M. A Frequency-Weighted Energy Operator and complementary ensemble empirical mode decomposition for bearing fault detection. Mech. Syst. Signal Process. 2017, 82, 103-116. [CrossRef]

32. Sun, P.; Liao, Y.; Lin, J. The shock pulse index and its application in the fault diagnosis of rolling element bearings. Sensors 2017, 17, 535. [CrossRef] [PubMed]

33. Ming, A.B.; Qin, Z.Y.; Zhang, W.; Chu, F.L. Spectrum auto-correlation analysis and its application to fault diagnosis of rolling element bearings. Mech. Syst. Signal Process 2013, 41, 141-154. [CrossRef]

34. Zhao, H.; Li, L. Fault diagnosis of wind turbine bearing based on variational mode decomposition and Teager energy operator. IET Renew. Power Gen. 2016, 11, 453-460. [CrossRef]

35. Rodriguez, P.H.; Alonso, J.B.; Ferrer, M.A.; Travieso, C.M. Application of the Teager-Kaiser energy operator in bearing fault diagnosis. ISA Trans. 2013, 52, 278-284. [CrossRef] [PubMed]

36. Pineda-Sanchez, M.; Puche-Panadero, R.; Riera-Guasp, M.; Perez-Cruz, J.; Roger-Folch, J.; Pons-Llinares, J.; Antonino-Daviu, J.A. Application of the Teager-Kaiser energy operator to the fault diagnosis of induction motors. IEEE Trans. Energy Conver. 2013, 28, 1036-1044. [CrossRef]

37. Lou, X.; Loparo, K.A. Bearing fault diagnosis based on wavelet transform and fuzzy inference. Mech. Syst. Signal Process 2004, 18, 1077-1095. [CrossRef]

38. Lee, J.; Qiu, H.; Yu, G.; Lin, J. Bearing Data Set, IMS. In Rexnord Technical Services; NASA Ames Prognostics Data Repository; University of Cincinnati: Cincinnati, OH, USA, 2007.

(C) 2017 by the authors. Licensee MDPI, Basel, Switzerland. This article is an open access article distributed under the terms and conditions of the Creative Commons Attribution (CC BY) license (http://creativecommons.org/licenses/by/4.0/). 